\title{
Biologia floral, melitofilia e influência de besouros Curculionidae no sucesso reprodutivo de Grobya amherstiae Lindl. (Orchidaceae: Cyrtopodiinae)
}

\author{
LUDMILA MICKELIUNAS $^{1,2}$, EMERSON R. PANSARIN ${ }^{1}$ e MARLIES SAZIMA ${ }^{1}$
}

(recebido: 10 de fevereiro de 2005; aceito: 27 de abril de 2006)

\begin{abstract}
Floral biology, melittophily and influence of curculionid beetles on the reproductive success of Grobya amherstiae Lindl. (Orchidaceae: Cyrtopodiinae)). The phenology, floral morphology, pollination mechanisms and reproductive biology of Grobya amherstiae Lindl. were studied in two populations located in altitudinal forests at Serra do Japi, Jundiaí, São Paulo State, Brazil. The flowering occurs in summer and lasts about one month (part of February and March). The flowers of an inflorescence open almost simultaneously, in the morning, and each lasts about seven to eight days. The flowers release a honey-like fragrance. At both populations G. amherstiae was pollinated by Paratetrapedia fervida Smith (Anthophoridae) bees, which collect floral oils produced by trichomatic elaiophores at the apex of the lip and the column basis. At one of the populations, besides bees individuals of a Curculionid beetle of the genus Montella were recorded, which perform selfpollination on the majority of the flowers. Grobya amherstiae is self-compatible but pollinator dependent. The females of Montella sp. oviposit in the ovary and their larvae consume the seeds. However, the number of fruits parasitized by the larvae is low when compared with the amount of fruits produced. Since natural fruit set is low, the beetles contribute positively for the reproductive success of $G$. amherstiae at least in one of the populations.
\end{abstract}

Key words - floral biology, Grobya, Orchidaceae, pollination, reproductive biology

RESUMO - (Biologia floral, melitofilia e influência de besouros Curculionidae no sucesso reprodutivo de Grobya amherstiae Lindl. (Orchidaceae: Cyrtopodiinae)). A fenologia, morfologia floral, mecanismos de polinização, biologia reprodutiva e sucesso reprodutivo de Grobya amherstiae Lindl. foram estudados em duas populações localizadas em regiões de mata mesofítica semidecídua de altitude da Serra do Japi (Jundiaí-SP). Grobya amherstiae floresce no verão, entre os meses de fevereiro e março. Todas as flores da inflorescência abrem mais ou menos simultaneamente pela manhã, e cada flor dura cerca de sete a oito dias. As flores emitem suave fragrância adocicada semelhante a do mel. Em ambas as populações G. amherstiae foi polinizada por abelhas Paratetrapedia fervida Smith (Anthophoridae), que coletam óleo produzido em elaióforos tricomáticos do ápice do labelo e da base da coluna. Além de $P$. fervida, em uma das populações, uma espécie de besouro do gênero Montella (Curculionidae) realizou autopolinizações na maioria das flores. Grobya amherstiae é autocompatível, mas depende de polinizador para transferência de pólen. As fêmeas do besouro ovipõem no ovário e suas larvas se alimentam das sementes. O número de frutos parasitados pelas larvas é baixo em relação à quantidade de frutos produzidos. Como a taxa de frutificação em condições naturais é baixa, os besouros contribuem para o sucesso reprodutivo de G. amherstiae em uma das populações.

Palavras-chave - biologia floral, biologia reprodutiva, Grobya, Orchidaceae, polinização

\section{Introdução}

A subtribo Cyrtopodiinae (Orchidaceae: Epidendroideae: Cymbidieae) abrange cerca de 12 gêneros, incluindo Grobya Lindl. (Dressler 1993). Grobya apresenta cinco espécies distribuídas entre as regiões Sul, Sudeste e Nordeste do Brasil (Barros \& Lourenço 2004). Grobya amherstiae Lindl. possui a distribuição mais ampla dentre as cinco espécies (Barros \& Lourenço 2004), ocorrendo em regiões de mata

\footnotetext{
1. Universidade Estadual de Campinas, Instituto de Biologia, Departamento de Botânica, Caixa Postal 6109, 13083-970 Campinas, SP, Brasil.

2._Autor para correspondência: colax@pop.com.br
}

semidecídua e Atlântica da maior parte do Leste do Brasil (Morrison 1997).

A maior parte das orquídeas é polinizada por engodo (Ackerman 1986). Por outro lado, as orquídeas que oferecem recursos aos seus visitantes apresentam ampla variedade de recursos: néctar, pólen, fragrâncias, pseudopólen ou óleos florais (van der Pijl \& Dodson 1966). Em Orchidaceae, a produção de óleos florais como recurso foi estudada detalhadamente por Vogel (1974) para algumas espécies das subtribos Ornithocephalinae e Oncidiinae. Segundo estudos mais recentes sobre a polinização de espécies de Oncidium desenvolvidos por Schlindwein (1995) e Singer \& Cocucci (1999), o óleo é coletado por abelhas do gênero Tetrapedia (Anthophoridae: Tetrapediini). Abelhas Anthophoridae (e.g. Centridini, Exomalopsini e Tetrapediini) utilizam óleos florais como alimento para 
suas larvas ou na construção de ninhos (Simpson \& Neff 1981, Roubik 1992).

A grande maioria das orquídeas é autocompatível (van der Pijl \& Dodson 1966). Autopolinização espontânea, no entanto, tende a ser evitada pela presença de mecanismos florais (van der Pijl \& Dodson 1966, Catling \& Catling 1991a). Polinizações manuais realizadas em condições experimentais promovem aumento na produção de frutos em muitas espécies de Orchidaceae quando comparado com as populações em ambiente natural (Janzen et al. 1980, Schemske 1980, Ackerman \& Oliver 1985, Montalvo \& Ackerman 1987, Zimmerman \& Aide 1989, Ackerman \& Montalvo 1990, Calvo 1990, Pansarin 2003). Em condições naturais, alguns fatores, como herbivoria (Stephenson 1981, Marquis 1984, Elmqvist et al. 1987, Snow \& Stanton 1988) e predação de frutos e sementes (Boucher \& Sork 1979, Arnold 1982, Haddock \& Chaplin 1982), podem influenciar negativamente no sucesso reprodutivo de espécies de orquídeas. Besouros da família Curculionideae podem parasitar ovários de algumas espécies dos gêneros Laelia, Cattleya (Monte 1942) e Oncidium (Bondar 1948), onde colocam seus ovos. As larvas se desenvolvem alimentando-se do conteúdo do ovário (Monte 1942).

Embora seja crescente o número de estudos sobre biologia da polinização e reprodução em Orchidaceae nos últimos anos no Brasil, ainda são muito escassos quando comparados à importância e ao número de espécies na família. Sobretudo, em relação aos gêneros sul-americanos e endêmicos do Brasil, como é o caso de Grobya, não existem informações sobre a biologia reprodutiva ou sobre os mecanismos de polinização de suas espécies. O presente trabalho tem por objetivo apresentar a biologia floral e reprodutiva de Grobya amherstiae Lindl., sua fenologia e morfologia floral, seus polinizadores, os mecanismos de polinização, assim como o sucesso reprodutivo em condições naturais, com destaque no comportamento do besouro Montella sp. e sua influência no sucesso reprodutivo de G. amherstiae.

\section{Material e métodos}

O estudo foi realizado na Serra do Japi (aproximadamente 2315' S e 4652’ W), no Município de Jundiaí - SP. A Serra do Japi é composta, predominantemente, de matas mesofíticas semidecíduas, florestas mesofíticas semidecíduas de altitude e esparsos enclaves de lajeados rochosos (Leitão Filho 1992). De acordo com Pinto (1992), a região é caracterizada por altitudes que variam entre 700 e 1.300 m e temperaturas médias anuais entre $15,7^{\circ} \mathrm{C}$ e $19,2^{\circ} \mathrm{C}$. A região apresenta sazonalidade bem marcada, com período de estiagem no outono e no inverno (abril a agosto) e períodos de chuvas mais intensas no verão (dezembro a março) (Pinto 1992).

O trabalho de campo foi realizado em duas populações de Grobya amherstiae Lindl. (aqui denominadas A e B) distantes cerca de $15 \mathrm{~km}$ uma da outra. A população A consta de cerca de 120 indivíduos, enquanto na área B ocorrem cerca de 500 plantas. Para acompanhamento da fenologia e biologia floral foram realizadas visitas quinzenais entre fevereiro de 2003 e agosto de 2004, sendo verificados: o período de produção de novos pseudobulbos, de inflorescências, aspectos sobre a biologia das flores e deiscência dos frutos.

Estudos sobre morfologia floral foram feitos com auxílio de estereomicroscópio binocular, a partir de flores frescas coletadas em ambas as populações.

As observações focais sobre os visitantes florais e para detecção dos mecanismos de polinização foram realizadas entre 3 e 9 de março de 2003 na população A, totalizando 23 horas e, entre 26 de fevereiro e 18 de março de 2004 em ambas as populações, perfazendo 65 horas, aproximadamente 30 horas na população A e 35 horas na B. O período de observação foi entre 7h00 e 18h00. Além disso, observações complementares foram realizadas ao longo do período de floração. Todas as observações foram feitas em diferentes condições de temperatura, precipitação e nebulosidade. Para detectar a possível ocorrência de polinizações noturnas, flores intactas foram marcadas ao final de cada dia de observação e verificadas quanto à remoção de polinários na manhã do dia seguinte.

A receptividade do estigma foi verificada em 30 flores intactas de diferentes idades (desde a antese até o préfenecimento) através do método de Galen \& Plowright (1977), que consiste em aplicar algumas gotas de $\mathrm{H}_{2} \mathrm{O}_{2}$ na região estigmática, que reage com efervescência enquanto estiver receptiva. A viabilidade do pólen foi testada de acordo com Radford et al. (1974), sendo utilizados 30 polinários. Cada polinário foi macerado individualmente sobre uma lâmina juntamente com uma gota de carmim acético e, em seguida, observado ao microscópio óptico. Para confirmar os resultados de viabilidade, foram realizados experimentos de germinação de pólen com 30 flores de diferentes idades, desde a antese até o pré-fenecimento, utilizando soluções de agaragar com três concentrações de sacarose: 10\%, 15\% e 20\% (Dafni 1992). Cada polinário ( $n=30$, sendo 10 para cada concentração) foi macerado em uma lâmina com uma gota de determinada solução de sacarose. Posteriormente, os grãos foram examinados em microscópio óptico, em intervalos regulares (de seis em seis horas), durante um período de 48 horas.

Para determinar o período que uma antera necessita para se desprender das polínias foram usados 30 polinários, coletados de 30 plantas diferentes. Cada polinário foi colocado em placa de petri imediatamente após sua remoção, tocado levemente com estilete até o desprendimento da antera 
e o período foi cronometrado. Todo o procedimento foi realizado em condições naturais, entre 8h30min e $9 \mathrm{~h} 00$ e temperatura entre 22 e $24^{\circ} \mathrm{C}$.

Os tratamentos envolvendo o sistema reprodutivo de Grobya amherstiae foram realizados no campo, a partir de inflorescências previamente ensacadas (Kearns \& Inouye 1993) com tule, utilizando um total de 28 indivíduos de ambas as populações. Foram realizados quatro tipos de tratamentos: autopolinização manual, autopolinização espontânea, emasculação e polinização cruzada. Apenas uma inflorescência de cada planta foi usada nessas polinizações, utilizando todas as flores no primeiro dia de antese.

As informações sobre o sucesso reprodutivo de Grobya amherstiae, em condições naturais, foram obtidas na população B em agosto de 2003 e nas populações A e B em agosto de 2004. Para cada população foram amostradas 30 inflorescências de diferentes plantas, sendo utilizadas todas as flores de cada inflorescência. Os dados de taxa de frutificação foram obtidos a partir de frutos deiscentes, logo no início de abertura das valvas. Na população B também foi verificada a porcentagem de frutos parasitados, obtida a partir de 30 inflorescências (287 flores) de diferentes plantas.

Para cada fruto proveniente dos tratamentos experimentais e dos coletados aleatoriamente no campo (um fruto por planta) foram examinados os embriões de 200 sementes. As sementes foram mergulhadas em solução de 1\% de cloreto 2,3,5-trifeniltetrazolium para coloração do embrião e examinadas ao microscópio óptico (Lakon 1949). Para análise das sementes foram considerados dois estados: sementes com embrião (potencialmente viáveis) e sementes sem embrião (inviáveis). A avaliação dos embriões foi realizada em frutos deiscentes e não parasitados.

O material testemunho de Grobya amherstiae (L. Mickeliunas \& E.R. Pansarin 47) foi depositado no Herbário da Universidade Estadual de Campinas (UEC) e o material entomológico no Museu de História Natural da Universidade Estadual de Campinas (ZUEC).

\section{Resultados e Discussão}

Na Serra do Japi, Grobya amherstiae Lindl. ocorre exclusivamente em matas mesofíticas semidecíduas de altitude, entre 1.000 e $1.300 \mathrm{~m}$, desenvolvendo-se sobre troncos primários e secundários de árvores de pequeno a grande porte. Entre agosto e outubro as plantas iniciam o desenvolvimento de um ou mais pseudobulbos e no início de janeiro os pseudobulbos formados no ano anterior emitem uma ou duas inflorescências laterais. As inflorescências são pêndulas e produzem de três a 15 flores ressupinadas. O início da antese, em ambas as populações, ocorre em meados de fevereiro e o pico de floração cerca de uma semana após. O período de floração se estende até o final de março.
As flores de Grobya amherstiae são amareladas com numerosas manchas castanho-vináceas (figura 1). As sépalas (ca. 1,8 × 0,7 cm) são amareladas com vênulas longitudinais castanho-vináceas; a sépala dorsal é elíptica e as laterais são linear-lanceoladas, retorcidas, revolutas, unidas na base e formando uma estrutura curva e oval, na qual o labelo é apoiado quando é desarticulado. As pétalas $(c a .1,7 \times 1,3 \mathrm{~cm})$ são assimetricamente ovais e amareladas com manchas castanho-vináceas. O labelo (0,7-0,8 × 0,9-1 cm) é cordiforme, amarelo claro e articulado com a base da coluna. Na porção apical do labelo há um elaióforo tricomático, semicircular e de coloração alaranjada (figura 1). A coluna (ca. 1,3 cm compr.) é recurvada e na base há um elaióforo tricomático semelhante ao do ápice do labelo (figura 3). O estigma é cordiforme e mede cerca de 1,5 × 2,2 mm. A antera (ca. 2,2 × 1,6 mm) é branca e algumas vezes apresenta uma mancha rósea no ápice. O polinário é composto por duas polínias ovais, unidas a um estipe translúcido e o viscídio terminal. As flores emitem odor adocicado que se assemelha ao do mel, principalmente nas horas mais quentes do dia. Em outras regiões as populações de G. amherstiae apresentam odor semelhante (Miller \& Warren 1996). Para mais detalhes sobre a morfologia de G. amherstiae veja Hoehne (1949) e Barros \& Lourenço (2004).

Em Grobya amherstiae a maioria das flores de cada inflorescência abriu mais ou menos simultaneamente, entre $6 \mathrm{~h} 00$ e 8h30min, com exceção de um ou dois botões apicais que abriram na manhã do dia seguinte. Cada flor durou cerca de sete a oito dias, mas quando o polinário foi removido a flor feneceu após dois a três dias. O estigma permaneceu receptivo desde a antese até o pré-fenecimento das flores. $O$ índice de grãos de pólen potencialmente viáveis, ao longo da vida da flor, foi de $100 \%$, no entanto, não houve germinação dos grãos de pólen em nenhuma das concentrações de sacarose, fato semelhante ao que ocorre em algumas espécies de Bulbophyllum (Borba et al. 1999).

Os óleos florais em Grobya amherstiae, produzidos tanto nos elaióforos da base da coluna quanto no ápice do labelo, estiveram disponíveis aos polinizadores desde a antese floral. Entretanto, após a retirada do óleo não ocorreu produção subseqüente dessa substância. Em Orchidaceae a oferta de óleos florais como recurso tem sido registrado em Oncidiinae (Vogel 1974, 1990, Schlindwein 1995, Singer \& Cocucci 1999) e Ornithocephalinae (Vogel 1974, 1990), entretanto, para Cyrtopodiinae este é o primeiro registro.

Os visitantes florais de Grobya amherstiae foram abelhas Paratetrapedia fervida Smith (Anthophoridae: 
Exomalopsini) em ambas as populações, e uma espécie de besouro do gênero Montella (Curculionidae: Centrinini) apenas na população B. Em ambas as populações de Grobya amherstiae a freqüência de Paratetrapedia fervida foi baixa (cerca de 3 a 4 registros por dia) e as visitas ocorreram em intervalos irregulares entre $9 \mathrm{~h} 30 \mathrm{~min}$ e $14 \mathrm{~h} 00$. As abelhas geralmente visitaram uma a várias flores de cada inflorescência, permanecendo cerca de 20 a 35 segundos em cada flor. Na população A as visitas ocorreram principalmente no final da floração, enquanto na área B as visitas foram mais freqüentes no início da florada. As visitas foram realizadas apenas em dias ensolarados e quentes, com temperaturas acima de $20^{\circ} \mathrm{C}$. A baixa freqüência de visitas pode estar relacionada ao clima instável, comum em áreas de altitude. Pequenas espécies de abelhas Centridini que habitam regiões neotropicais geralmente não forrageiam sob condições adversas, como nevoeiros, ventos fortes, chuvas intensas e baixas temperaturas (Roubik 1992).

A visita de Paratetrapedia fervida às flores de Grobya amherstiae iniciou-se com o pouso da abelha sobre a face inferior do labelo, que em seguida se dirigiu para o elaióforo ou, em outras ocasiões, a abelha pousou diretamente sobre o elaióforo. Para obter o óleo a abelha apoiou-se com as pernas traseiras na face inferior do labelo, recolheu o óleo com as pernas dianteiras e medianas (figura 1) e transferiu-o para a porção ventral do tórax. Após visitar várias flores, a abelha transferiu o óleo do tórax para as escopas nas pernas traseiras. Durante a atividade de recolher óleo a abelha podia desarticular ou não o labelo. Quando o labelo era desarticulado (figura 2) a abelha dirigia-se para o elaióforo da base da coluna. Com esse deslocamento da abelha, o labelo era articulado novamente, arremessando-a contra a coluna (figura 3). Ao abandonar a flor, a abelha contatava o viscídio com o escutelo, de modo que o polinário, juntamente com a antera, era removido (figura 4). Abelhas Anthophoridae são conhecidas como coletoras de óleos florais atuando como polinizadoras em espécies de diversas famílias (Vogel 1974, Simpson \& Neff 1981, Roubik 1992), incluindo algumas espécies de orquídeas (Vogel 1974, Schlindwein 1995, Singer \& Cocucci 1999). O comportamento de coleta de óleo de $P$. fervida em
G. amherstiae, assemelha-se ao de outras Anthophoridae em flores de orquídeas (Vogel 1974, Schlindwein 1995, Singer \& Cocucci 1999). Entretanto, o mecanismo de polinização que compreende o arremesso da abelha em direção à coluna devido ao processo de desarticulação/articulação do labelo, conhecido em espécies de Pleurothallis e Bulbophyllum que produzem néctar (Borba \& Semir 1998, 2001), ou são polinizadas por engano (Borba \& Semir 2001), não havia sido documentado em orquídeas que oferecem óleos florais como recurso aos polinizadores.

Em Grobya amherstiae a antera demorou cerca de 10 a 15 minutos para se desprender naturalmente do polinário, período maior que o da permanência da abelha em uma inflorescência (20 segundos a 3 minutos). Somente após esse período as polínias poderiam ser depositadas no estigma. Esta característica tende a evitar a deposição do polinário em flores da mesma inflorescência, o que favorece a polinização cruzada. Em Orchidaceae a autopolinização é evitada, principalmente, através de mecanismos florais (van der Pijl \& Dodson 1966, Catling \& Catling 1991a). A retenção do capuz da antera pelo polinário, prevenindo autopolinização, é um mecanismo muito difundido, sendo registrado em outros gêneros na família (Catling \& Catling 1991b, Singer \& Cocucci 1999, Borba \& Semir 2001).

Os besouros Montella sp. ocorreram desde o início até o final do período de floração. Geralmente houve um a quatro indivíduos por inflorescência. Ao atingir a coluna o besouro deslocou a antera e consumiu as paredes que separam as polínias. Após isto, provavelmente, à procura de outras substâncias comestíveis (viscídio, por exemplo), o besouro deslocou o polinário depositando-o no estigma (figura 5), onde continuou manipulando o polinário por cerca de 10 a 20 minutos (figura 6). Esse comportamento foi repetido em todas as flores das inflorescências, resultando em 100\% de autopolinizações e posterior desenvolvimento de frutos. Até o momento esse comportamento de Montella sp. não havia sido documentado em orquídeas. A interação entre Montella sp. e Grobya amherstiae pode ser comparada às interações que ocorrem em espécies de Yucca (Agavaceae) e Ficus (Moraceae), polinizadas

Figures 1-6. Flowers of Grobya amherstiae Lindl. and its pollinators. 1. Paratetrapedia fervida Smith collecting floral oils of the elaiophore at the lip apex (arrow). 2. Disarticulating the lip. 3. Entering the flower after lip articulation to access the elaiophore at the column basis (arrow). 4. Leaving the flower after pollinarium removal. Note the pollinarium with the anther on the bee scutellum. 5. Montella sp. displacing the anther. 6. Placing the pollinarium on the stigma. Bars $=5 \mathrm{~mm}$. 

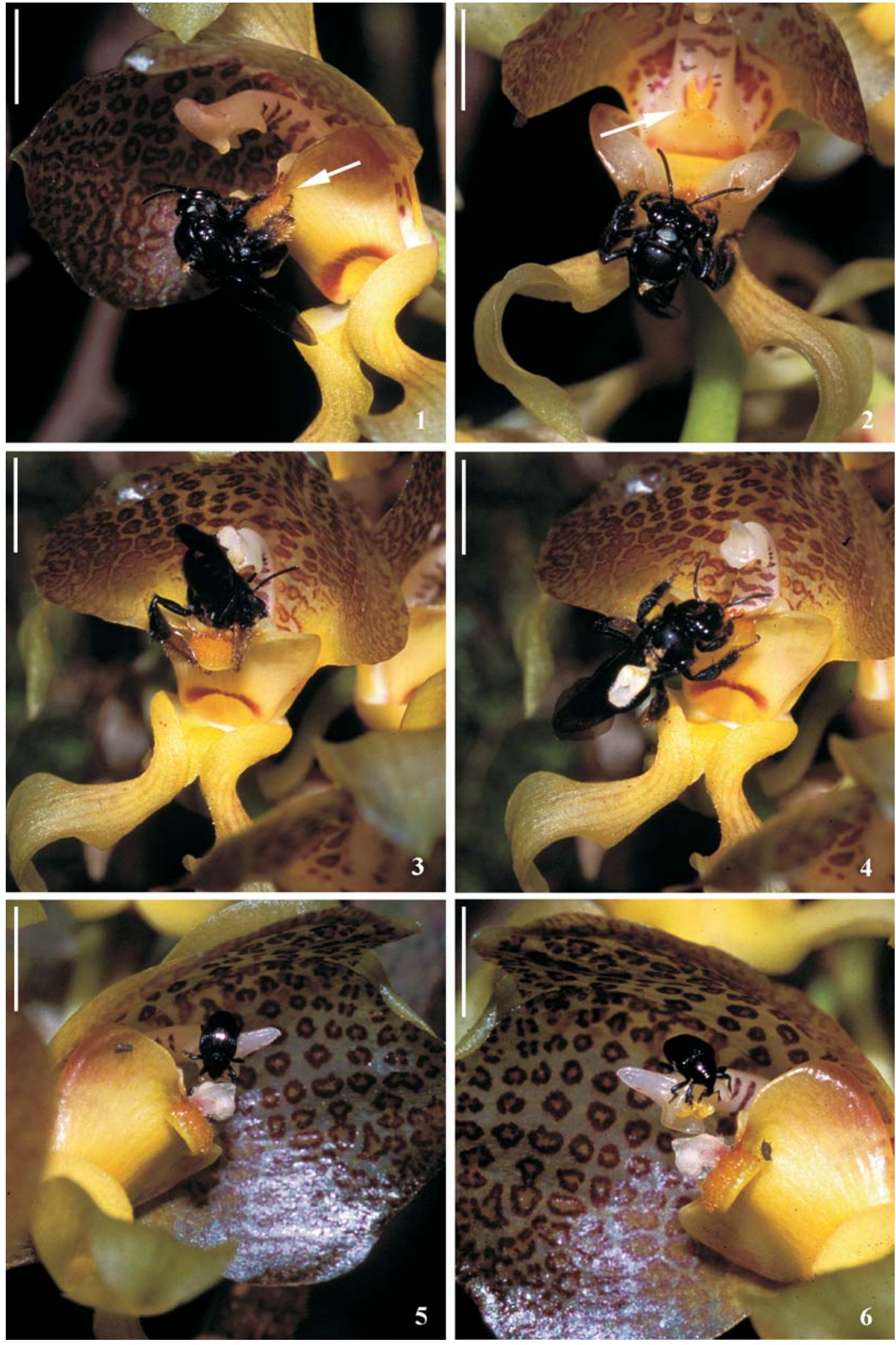

Figuras 1-6. Flores de Grobya amherstiae Lindl. e seus polinizadores. 1. Paratetrapedia fervida Smith recolhendo óleo do elaióforo do ápice do labelo (seta). 2. Desarticulando o labelo (seta). 3. Entrando na flor após articulação do labelo para acessar o elaióforo da base da coluna. 4. Abandonando a flor após remoção do polinário. Note polinário depositado, juntamente com a antera, no escutelo da abelha. 5. Montella sp. deslocando a antera da flor. 6. Depositando o polinário no estigma da flor. Barras $=5 \mathrm{~mm}$. 
por mariposas e vespas, respectivamente (revisão em Ramírez 1974, Herre 1989, Compton et al. 1996, Machado et al. 1996 e Pellmyr 2003). Polinização por besouros, embora não por curculionídeos, tem sido registrada para outros gêneros de Orchidaceae. Nesses casos, no entanto, os besouros são atraídos por engano ou para obter néctar das flores (van der Pijl \& Dodson 1966, Singer \& Cocucci 1997).

Grobya amherstiae é autocompatível, uma vez que se desenvolveram frutos a partir das autopolinizações manuais (tabela 1). Não houve formação de frutos em flores emasculadas ou através de autopolinizações espontâneas, mas em autopolinizações manuais e em polinizações cruzadas a porcentagem de frutos desenvolvidos foi de 55,8\% e 82,0\%, respectivamente (tabela 1). Autocompatibilidade é comum em Orchidaceae, e as barreiras mecânicas da flor normalmente são eficientes para evitar autopolinizações (van der Pijl \& Dodson 1966, Catling \& Catling 1991a).

Os frutos tornaram-se deiscentes cerca de 170 a 180 dias após as polinizações. A porcentagem de sementes potencialmente viáveis em frutos originados das autopolinizações manuais (99,35\%) não apresentou diferenças significativas em relação aos formados através de polinizações cruzadas $(99,36 \%)$, nem tampouco em relação aos provenientes de condições naturais, com 99,6\% e 99,7\% nas populações A e B, respectivamente (tabela 1 ). $O$ alto percentual de sementes viáveis em Grobya amherstiae não é comum em Epidendroideae, que geralmente apresenta espécies com alto número de sementes sem embrião (Lock \& Profita 1975, Stort \& Martins 1980, Stort \& Galdino 1984, Borba et al. 2001, Borba \& Braga 2003). Outras Epidendroideae, como Xylobium squalens (Lindl.)
Lindl. (Maxillarieae), por exemplo, também produzem alto número de sementes viáveis (Pintaúdi et al. 1990).

Em condições naturais a taxa de frutificação da população A, onde apenas Paratetrapedia fervida atuou como polinizador, foi inferior a $8 \%$, ao passo que na população $\mathrm{B}$, onde também ocorreram os besouros, essa taxa ultrapassou 50\% (tabela 1). A taxa de frutificação na população $\mathrm{B}$ é semelhante à registrada para outras espécies de orquídeas polinizadas por besouros (Singer \& Cocucci 1997). Portanto, é expressiva a influência de Montella sp. sobre o sucesso reprodutivo de G. amherstiae.

Na população B, cerca de $5 \%$ dos frutos foram parasitados por larvas do besouro Montella sp., que se alimentam do conteúdo do ovário, à semelhança de espécies de besouros curculionídeos do gênero Diorymerellus, que ovipositam em flores de algumas espécies de orquídeas, utilizando-as para completar seu ciclo de vida (Monte 1942). Embora Monte (1942) não tenha registrado a atividade desses curculionídeos como polinizadores é bastante provável que esses casos sejam semelhantes ao de Grobya amherstiae.

A porcentagem de frutos parasitados (5\%) foi pequena em relação às polinizações efetuadas pelos besouros (acima de 50\%, tabela 1). Portanto, apesar do parasitismo, a atividade dos besouros foi vantajosa para o sucesso reprodutivo de G. amherstiae. Tendo em vista que Paratetrapedia fervida é pouco freqüente e os frutos formados por essas abelhas são escassos, a atividade de Montella sp. como polinizador pode ser um fator importante para a reprodução de G. amherstiae, podendo refletir sobre o tamanho de suas populações. Escassez de frutos é comum em muitas espécies de orquídeas não autógamas e está relacionada,

Tabela 1. Resultados dos testes sobre o sistema de reprodução de Grobya amherstiae Lindl.: porcentagens de frutificação e de sementes potencialmente viáveis em cada um dos tratamentos realizados e em condições naturais nas duas populações (A e B). Entre parênteses estão os números de frutos/flores e número de sementes viáveis/sementes.

Table 1. Results of the breeding system of Grobya amherstiae Lindl.: fruit set and proportion of viable seeds per treatment in natural conditions of the two populations (A and B). In parentheses are number of fruits/flowers and viable seeds/seeds.

\begin{tabular}{lcc}
\hline Tratamentos & Frutificação & Sementes \\
\hline Autopolinização manual & $55,8 \%(37 / 64)$ & $99,35 \%(7352 / 7400)$ \\
Polinização cruzada & $82 \%(41 / 50)$ & $99,36 \%(8148 / 8200)$ \\
Emasculação & $0(0 / 51)$ & - \\
Autopolinização espontânea & $0(0 / 55)$ & - \\
Condições naturais (A) & \\
Condições naturais (B) ${ }^{2}$ & $7,9 \%(23 / 300)$ & $99,6 \%(3984 / 4000)$ \\
\hline
\end{tabular}

${ }^{1}$ Apenas abelhas/only bees; ${ }^{2}$ Abelhas e besouros/bees and beetles. 
principalmente, com a transferência de pólen deficiente entre indivíduos, na qual a escassez de polinizadores parece ser o fator limitante (Janzen et al. 1980, Schemske 1980, Ackerman \& Oliver 1985, Montalvo \& Ackerman 1987, Zimmerman \& Aide 1989, Ackerman \& Montalvo 1990, Calvo 1990) e na Serra do Japi é o principal fator responsável pelo baixo sucesso reprodutivo de várias espécies de orquídeas (Pansarin 2000, 2003).

Agradecimentos - Ao CNPq, pelo auxílio financeiro; à Fapesp, pelo apoio através do trabalho de Iniciação Científica de L. Mickeliunas (processo 03/05383-3), junto ao Curso de Graduação em Ciências Biológicas da Universidade Estadual de Campinas; a Fábio de Barros e Ricardo A. Lourenço, pelas sugestões no desenvolvimento do projeto; a Isabel Alves dos Santos e Antonio José Camilo Aguiar, pela identificação de Paratetrapedia; a Sérgio Vanin, do Departamento de Zoologia da USP, pela identificação do Cucurlionidae; a Ariadna Valentina Lopes e um assessor anônimo, pelas valiosas sugestões; à Base Ecológica da Serra do Japi e à Guarda Municipal de Jundiaí, pela autorização para realização dos trabalhos de campo.

\section{Referências bibliográficas}

ACKERMAN, J.D. 1986. Mechanisms and evolution of fooddeceptive pollination systems in orchids. Lindleyana 1:108-113.

ACKERMAN, J.D. \& MONTALVO, A.M. 1990. Short-and long-term limitations to fruit production in a tropical orchid. Ecology 71:263-272.

ACKERMAN, J.D. \& OLIVER, J.C. 1985. Reproductive biology of Oncidium variegatum: moon phases, pollination, and fruit set. American Orchid Society Bulletin 54:326-329.

ARNOLD, R.M. 1982. Pollination, predation and seed set in Linaria vulgaris (Scrophulariaceae). American Midland Naturalist 107:360-369.

BARROS, F. \& LOURENÇO, R.A. 2004. Synopsis of the Brazilian orchid genus Grobya, with the description of two new species. Botanical Journal of the Linnean Society 145:119-127.

BONDAR, G. 1948. Notas entomológicas da Bahia. XX. Revista de Entomologia 19:1-54.

BORBA, E.L. \& BRAGA, P.I.S. 2003. Biologia reprodutiva de Pseudolaelia corcovadensis (Orchidaceae): melitofilia e autocompatibilidade em uma Laeliinae basal. Revista Brasileira de Botânica 26:541-549.

BORBA, E.L., SHEPHERD, G.J. \& SEMIR, J. 1999. Reproductive systems and crossing potential in three species of Bulbophyllum (Orchidaceae) occurring in Brazilian “campo rupestre" vegetation. Plant Systematics and Evolution 217:205-214.
BORBA, E.L., SHEPHERD, G.J. \& SEMIR, J. 2001. Selfincompatibility, inbreeding depression and crossing potential in five Pleurothallis (Orchidaceae) species. Annals of Botany 88:89-99.

BOUCHER, D.H. \& SORK, V.L. 1979. Early drop of nuts in response to insect infestation. Oikos 33:440-443.

CATLING, P.M. \& CATLING, V.R. 1991a. A synopsis of breeding systems and pollination in North American orchids. Lindleyana 6:187-210.

CATLING, P.M. \& CATLING, V.R. 1991b. Anther cap retention in Tipularia discolor. Lindleyana 6:113-116.

COMPTON, S.G., WIEBES, J.T. \& BERG, C.C. 1996. The biology of fig trees and their associated animals. Journal of Biogeography 23:405-407.

DAFNI, A. 1992. Pollination ecology: a practical approach, Oxford University Press, Oxford.

DRESSLER, R.L. 1993. Phylogeny and classification of the orchid family. Cambridge University Press, Cambridge.

ELMQVIST, T., ERICSON, L., DANELL, K. \& SALOMONSON, A. 1987. Flowering, shoot production, and vole bark herbivory in a boreal willow. Ecology 68:1623-1629.

GALEN, C. \& PLOWRIGHT, R.C. 1977. Testing the accuracy of using peroxidase activity to indicate stigma receptivity. Canadian Journal of Botany 65:107-111.

HADDOCK, R.C. \& CHAPLIN, S.J. 1982. Pollination and seed production in two phenologically divergent prairie legumes (Baptisia leucophaea and B. eucantha) American Midland Naturalist 108:175-186.

HERRE, E.A. 1989. Coevolution and reproductive characteristics in twelve species of new word figs and their pollinator wasps. Experiencia 45:637-647.

HOEHNE, F.C. 1949. Iconografia de orquidáceas do Brasil. Instituto de Botânica, São Paulo.

JANZEN, D.H., DEVRIES, P., GLADSTONE, D.E., HIGGINS, M.L. \& LEWINSOHN, T.M. 1980. Self-and crosspollination of Encyclia cordigera (Orchidaceae) in Santa Rosa National Park, Costa Rica. Biotropica 12:72-74.

KEARNS, C. \& INOUYE, W. 1993. Techniques for pollination biologists. University Press of Colorado, Niwot.

LAKON, G. 1949. The topographical tetrazolium method for determining the germination capacity of seeds. Plant Physiology 24:389-394.

LEITÃO FILHO, H.F. 1992. A flora arbórea da Serra do Japi. História natural da Serra do Japi. In (L.P.C. Morellato, ed.). Editora da Unicamp/Fapesp, Campinas, p.40-62.

LOCK, J.M. \& PROFITA, J.C. 1975. Pollination of Eulophia cristata (Sw.) Steud. (Orchidaceae) in Southern Ghana. Acta Botanica Neerlandica 24:135-138.

MACHADO, C.A., HERRE, E.A., MCCAFFERTY, S. \& BERMINGHAM, E. 1996. Molecular phylogenyes of fig pollinating and nonpollinating wasps and the implication of the origin and evolution of the fig wasp mutualism. Journal of Biogeography 23:531-542.

MARQUIS, R.J. 1984. Leaf herbivores decrease fitness of a tropical plant. Science 226:537-539. 
MILLER, D. \& WARREN, R. 1996. Orquídeas do alto da Serra, $2^{a}$ ed. Salamandra, Rio de Janeiro.

MONTALVO, A.M. \& ACKERMAN, J.D. 1987. Limitations to fruit production in Ionopsis utricularioides (Orchidaceae). Biotropica 19:24-31.

MONTE, O. 1942. Duas novas espécies de “Diorymerellus” (Col. Curculionidae), prejudiciais às orquídeas. Arquivos do Instituto Biológico 13:87-91.

MORRISON, A. 1997. Grobya. In The illustrated encyclopedia of orchids (A. Pridgeon, ed.). Timber Press, Oregon, p.137-138.

PANSARIN, E.R. 2000. Biologia reprodutiva e morfologia floral de espécies de Orchidaceae em diferentes ambientes no Estado de São Paulo. Dissertação de mestrado, Universidade Estadual de Campinas, Campinas.

PANSARIN, E.R. 2003. Biologia reprodutiva e polinização em Epidendrum paniculatum Ruíz \& Pavón (Orchidaceae). Revista Brasileira de Botânica 26:203-211.

PELLMYR, O. 2003. Yuccas, yucca moths, and coevolution: a review. Annals of the Missouri Botanical Garden 90:35-55

PINTAÚDI, C.A., STORT, M.N.S. \& MARIN-MORALES, M.A. 1990. Polinizações naturais e artificiais de Xylobium squalens Lindl. (Orchidaceae). Naturalia 15:67-80.

PINTO, H.S. 1992. O clima da Serra do Japi. In História natural da Serra do Japi (L.P.C. Morellato, ed.). Editora da Unicamp/Fapesp, Campinas, São Paulo, p.30-38.

RADFORD, A.E., DICKINSON, W.C., MASSEY, J.R. \& BELL, C.R. 1974. Vascular plant systematics. Harper \& Row, New York.

RAMÍREZ, B.W. 1974. Coevolution of Ficus and Agaonidae. Annals of the Missouri Botanical Garden 61:770-780.

ROUBIK, D.W. 1992. Ecology and natural history of tropical bees. Cambridge University Press, Cambridge.

SCHEMSKE, D.W. 1980. Evolution of floral display in the orchid Brassavola nodosa. Evolution 34:489-493.
SCHLINDWEIN, C. 1995. Wildbienen und ihre Trachtpflanzen in einer sudbrasilianischen Buschlandschaft: Fallstudie Guaritas, Bestäubung bei Kakteen und Loasaceen. Verlag Ulrich E. Grauer, Stuttgart.

SIMPSON, B.B. \& NEFF, J.L. 1981. Floral rewards: alternatives to pollen and nectar. Annals of the Missouri Botanical Garden 68:301-322.

SINGER, R.B. \& COCUCCI, A.A. 1997. Pollination of Pteroglossaspis ruwenzoriensis (Rendle) Rolfe (Orchidaceae) by beetles in Argentina. Botanica Acta 110:338-342.

SINGER, R.B. \& COCUCCI, A.A. 1999. Pollination mechanisms in four sympatric southern Brazilian Epidendroideae orchids. Lindleyana 14:47-56.

SNOW, A.A. \& STANTON, M.L. 1988. Aphids limit fecundity of a weedy annual (Raphannus sativus). American Journal of Botany 75:589-593.

STEPHENSON,A.G. 1981. Flower and fruit abortion: proximate causes and ultimate functions. Annual Review of Ecology and Systematics 12:253-279.

STORT, M.N.S. \& GALDINO, G.L. 1984. Self- and crosspollination in some species of the genus Laelia Lindl. (Orchidaceae). Revista Brasileira de Genética 7:671-676.

STORT, M.N.S. \& MARTINS, P.S. 1980. Autopolinização e polinização cruzada em algumas espécies do gênero Cattleya (Orchidaceae). Ciência e Cultura 32:1080-1084.

VAN DER PIJL, L. \& DODSON, C.H. 1966. Orchid flowers, their pollination and evolution. University of Miami Press, Coral Gables.

VOGEL, S. 1974. Ölblumen und ölsammelnde Bienen. Akademie der Wissenschaften und der Literatur Tropische und subtropische Pflanzenwelt 7:1-267.

VOGEL, S. 1990. History of Malpighiaceae in the light of pollination ecology. Memoirs of the New York Botanical Garden 55:130-142.

ZIMMERMAN, J.K. \& AIDE, M. 1989. Patterns of fruit production in a Neotropical orchid: pollinator vs. resource limitation. American Journal of Botany 76:67-73. 